\title{
Analysis of Related Factors Influencing the Efficacy of Extracorporeal Shockwave in the Treatment of Fracture Nonunion: A Prospective Study in Shanghai, China
}

\section{Ang Li}

Shanghai Jiao Tong University Affiliated Sixth People's Hospital

\section{Rui Wang}

Shanghai Jiao Tong University Affiliated Sixth People's Hospital

\section{Shiyang Yu}

Shanghai Jiao Tong University Affiliated Sixth People's Hospital Jinghuan Huang

Shanghai Jiao Tong University Affiliated Sixth People's Hospital

\section{Lixin Jiang}

Shanghai Jiao Tong University Affiliated Sixth People's Hospital

Xiaolin Li ( $\square$ lixiaolin@sjtu.edu.cn )

Shanghai Jiao Tong University Affiliated Sixth People's Hospital

\section{Research Article}

Keywords: Extracorporeal shockwave therapy, fracture nonunion, ultrasound examination

Posted Date: September 28th, 2021

DOI: https://doi.org/10.21203/rs.3.rs-930135/v1

License: (c) (1) This work is licensed under a Creative Commons Attribution 4.0 International License.

Read Full License 


\section{Abstract}

Background: Extracorporeal shockwave is recommended as the first choice for the treatment of fracture nonunion, However, some patients with fracture nonunion have poor response to extracorporeal shockwave therapy. The present study aimed to investigate related factors which may affect the clinical efficacy of extracorporeal shockwave on fracture nonunion.

Methods: Ultrasound examination was applied to observe nonunion gap, local blood supply, hardness of callus and hematoma formation before and after extracorporeal shockwave therapy. The patient's condition was followed up for 12 months after extracorporeal shockwave therapy. Four correlations and regression analysis methods were applied to analyze the factors which were correlated the clinical efficacy of extracorporeal shockwave. Receiver-operating characteristic analysis revealed that the cut-off of the fracture nonunion gap was $4.200 \mathrm{~mm}$ and the cut-off of the hardness of callus/impulse energy was 2.555. Single factor linear correlation analysis, multi-factor linear regression analysis, single-factor logistic regression analysis were applied.

Results: The four ultrasound signs were significantly correlated with extracorporeal shockwave therapy: nonunion gap $(r=-0.723 ; O R=3.074)$, local blood supply $(r=0.611 ; O R=0.191)$, hardness of callus/impulse energy $(r=-0.510 ; O R=19.942)$, and hematoma formation $(r=0.722 ; O R=0.015)$.

Conclusions: Nonunion gap larger than $4.200 \mathrm{~mm}$ and hardness of callus/impulse energy larger than 2.555 are risk factors of extracorporeal shockwave therapy outcome, and good local blood supply and effective hematoma formation induced by extracorporeal shockwave therapy are protective factors. By analyzing the related factors of extracorporeal shockwave therapy on fracture nonunion, clinicians may screen out the patients with fracture nonunion who are not suitable for extracorporeal shockwave therapy, and reduce the risk of fracture nonunion.

\section{Background}

Nonunion of bone is the body's inability to heal a fracture. The most agreed-upon standard definition of nonunion made by the FDA is a fracture that persists for a minimum of nine months without signs of healing for three months[1]. Fracture nonunion increases the psychological pressure of patients and increases the economic burden of the family and society. Extracorporeal shockwave therapy (ESWT) has been widely used to treat many orthopedic diseases and proven to be effective and non-invasive[2-4]. Many studies have investigated the effects of extracorporeal shockwave on bone repair; and positive effects have been demonstrated in both acute fracture and fracture nonunion. Some studies report that ESWT has the same effect as surgical repair on fracture nonunion, and extracorporeal shockwave can be recommended as the first choice for the treatment of fracture nonunion[5-7].

However, it has also been found that some patients with fracture nonunion have poor response to ESWT [8]. The patients with fracture nonunion often need to be observed for 3-6 months or even more than 12 months after ESWT. For the patients who do not respond well, ESWT may delay surgical treatment, 
increase osteoporosis, and even risk internal fixation fractures, which may affect the effect of subsequent surgical bone grafting [9].

After more than 40 years of continuous development, ultrasound examination (UE) has provided more and more information and help for the clinical diagnosis and treatment of orthopedic diseases.

Musculoskeletal ultrasound has become one of the main clinical imaging diagnostic techniques like Xray, CT, and MRI for the musculoskeletal system[10].Moreover, compared with other imaging techniques, high-resolution musculoskeletal ultrasound is effective, inexpensive, and without radiation exposure in fracture assessment[11]. The present article discusses related factors influencing the effect of extracorporeal shockwave in the treatment of fracture nonunion, and provides a theoretical basis for the patients with fracture nonunion to choose appropriate treatment in time.

\section{Methods}

\section{Patient population}

Sixty-three nonunion patients (seventy-two bones) came to our hospital for treatment and met the inclusion criteria from January 2016 to December 2019. Among them, forty-three were males and twenty were females, with an average age of $41.4 \pm 14.0$ years old (range 11-76). The most common sites involved were the femur and tibia. The average time from initial fracture treatment to extracorporeal shockwave intervention was 12.6 months (9 months to 3.5 years). Inclusion criteria: All patients received external fixation or internal fixation after fracture. The fracture line was still clear on X-ray examination 9 months after the fracture, and there was no sign of healing. The coagulation test was normal, the local soft tissues were in good condition, without skin lesions or infections. Exclusion criteria: all patients whose clinical diagnosis did not meet the diagnostic criteria of nonunion; infectious nonunion, obvious hardening of the fractured end, pathological fracture, active osteomyelitis, wearing a pacemaker, skin injury, severe local scars, severe coagulation patients with dysfunction were excluded. All patients and their family were informed and signed a consent form. This study was formally approved by the ethics committee of Shanghai sixth people's hospital (Approval No:2016-023).

\section{Treatment of nonunion}

Shockwave orthopedic therapy device was applied to treat fracture nonunion (HK.SWT-200, Shenzhen Huikang Medical Equipment Co., Ltd. Shenzhen, China). ESWT procedures were performed according to the following principles: 1) X-ray combined with ultrasound was used to locate nonunion and to select points to treat; 2 ) the transfer form of shock wave source energy was focused, and each point received 2,000 impulses; 3 ) impulse energy was as high as patients could tolerate. Generally, the energy range was $0.26 \otimes 0.39 \mathrm{~mJ} / \mathrm{mm}^{2}$; 4) after the treatment of one impact point, checking whether the skin of the impact site was damaged. 5) treatment involved five sessions with intervals of one day between sessions; 6 ) adequate intakes of calcium and vitamin D after ESWT were ensured[3, 12, 13]. 


\section{Ultrasound examination and outcome measurements}

All patients underwent UE before and after ESWT using a 9L4 (4-9 MHz) transducer and an Acuson S3000 ultrasound system (Siemens Medical Solutions USA『lnc.). The initial ultrasound examination was performed before ESWT to observe the size of nonunion gap, blood supply of the nonunion area, and hardness of callus. After ESWT, ultrasound was performed again to observe the formation of subperiosteal hematoma in the nonunion area. The maximum value of the nonunion gap was selected and measured in millimeters $(\mathrm{mm})$. Local blood supply was divided into 1 to 4 grades (corresponding to poor, bad, good, and very good respectively) according to two designated doctor's experiences since there was no quantitative method available for this measurement. The two doctors had been specially trained before doing this work. Hardness of callus was measured by acoustic radiation force impulse (ARFI), and shear wave velocity[14]. However, considering the hardness was related to the extracorporeal shockwave impulse energy, we changed this sign to hardness of callus/impulse energy. The periosteal reaction was defined as periosteum hematoma formation after original ESWT and the ultrasound sign was the presence of a hypoechoic band or fluid dark area under the periosteum which we graded as 0 (absent) or 1 (present).

All patients were followed up for 12 months after ESWT. In order to provide an objective basis for judging the influence of extracorporeal shockwave on fracture nonunion, the fracture site was examined by $X$ ray (Discovery XR650 X-ray system, GE Healthcare, US) before and 12 months after ESWT. During the treatment, the unstable fracture end of the patient was immobilized, and other chronic diseases were treated [15]. By comparing the X-ray images before and 12 months after ESWT, areas of new healing could be observed and outcomes were graded according to the newly-healing area of nonunion: $0-20 \%$ healing area was defined as grade $0,20-40 \%$ as grade $1,40-60 \%$ as grade $2,60-80 \%$ as grade 3 , and $80-100 \%$ as grade 4 (see detail in supporting information: S. $1-$ S. 5 ).

The devices should be tested to ensure that it can be used normally, and sterilize the impact gun head, and prepare the required medium coupling agent.

\section{Statistical analysis}

All data were analyzed using SPSS 19.0 (IBM Corp., Armonk, NY, USA), with the level of significance set at $P<0.05$. Data analysis included descriptive statistics, single factor linear correlation analysis of the four ultrasound signs, multi-factor linear regression analysis, single-factor logistic regression analysis, and receiver-operating characteristic (ROC) testing to analyze the nonunion gap and hardness of callus/impulse energy. (Outcome was changed to good prognosis (healing grade 3,4 ) or bad prognosis (healing grade 1,2) for single-factor logistic regression analysis and ROC tests).

\section{Results}




\section{Fracture nonunion site and the effect of ESWT on fracture nonunion after 12 months}

Sixty-three patients, including seventy-two nonunion sites were included in our study. Seventy-two nonunion sites were observed, involving the femur $(n=26)$, tibia $(n=19)$, humerus $(n=10)$, neck of femur $(n=3)$, fibula $(n=7)$, ulna $(n=3)$, radius $(n=2)$, clavicle $(n=1)$, and metacarpus $(n=1)$. By comparing $X-$ ray images before and 12 months after ESWT, the healing of all fracture nonunion was found to be different. Among these nonunion, 19 achieved grade 4 healing (26\%), 23 achieved grade 3 healing (32\%), ten achieved grade 2 healing (14\%), 13 had grade 1 healing (18\%) and seven had grade 0 healing $(10 \%)$.

\section{The relationship between the four ultrasound signs and nonunion healing after ESWT}

The results of single-factor linear correlation analysis are shown in Table 1, single factor logistic regression analysis in Table 2 and multi-factor linear regression analysis is shown in Table 3.

Table 1

Single-factor linear regression analysis of ESWT outcome.

\begin{tabular}{|llll|}
\hline & Pearson & Sig. & $95 \% \mathrm{Cl}$ \\
\hline Gap of nonunion & -0.723 & 0.000 & $-0.447 \rrbracket-0.281$ \\
\hline Local blood supply & 0.611 & 0.000 & $0.558 \otimes 1.056$ \\
\hline Hardness of callus/impulse energy & -0.510 & 0.000 & $-2.023 \rrbracket-0.846$ \\
\hline Hematoma formation & 0.722 & 0.000 & $1.464 \rrbracket 2.331$ \\
\hline
\end{tabular}

Table 2

Single-factor logistic regression analysis of ESWT outcome.

\begin{tabular}{|llll|}
\hline & OR & Sig. & $\mathbf{9 5 \%} \mathrm{Cl}$ \\
\hline Gap of nonunion & 3.074 & 0.000 & $1.718 \rrbracket 5.500$ \\
\hline Local blood supply & 0.191 & 0.000 & $0.087 \rrbracket 0.419$ \\
\hline Hardness of callus/impulse energy & 19.942 & 0.000 & $4.385 \llbracket 90.69$ \\
\hline Hematoma formation & 0.015 & 0.000 & $0.003 \llbracket 0.069$ \\
\hline
\end{tabular}


Table 3

Multi-factor linear regression analysis of ESWT outcome.

\begin{tabular}{|llll|}
\hline & $\mathbf{t}$ & Sig. & $\mathbf{9 5 \% \mathrm { Cl }}$ \\
\hline Gap of nonunion & -5.224 & 0.000 & $-0.275 \rrbracket-0.123$ \\
\hline Local blood supply & 3.268 & 0.002 & $0.123 \rrbracket 0.509$ \\
\hline Hardness of callus/impulse energy & -2.056 & 0.044 & $-0.795 \rrbracket-0.012$ \\
\hline Hematoma formation & 4.288 & 0.000 & $0.477 \rrbracket 1.307$ \\
\hline
\end{tabular}

Then, ROC analysis revealed that cut-off value of the fracture nonunion gap was $4.200 \mathrm{~mm}$ and the hardness of callus/impulse energy was 2.555 , respectively (Fig. 1). The ultrasound result of nonunion gap measuring showed that the diameters of the defects directly (Fig. 2). The ultrasound result for local blood supply detecting showed the grade of blood supply of callus in each patient (Fig. 3). The ARFI measured the hardness of callus clearly (Fig. 4). Ultrasound detection showed hematoma (Fig. 5). These four ultrasound signs were significantly correlated with extracorporeal shockwave therapy: nonunion gap $(r=-0.723 ; O R=3.074)$, local blood supply $(r=0.611 ; O R=0.191)$, hardness of callus/impulse energy $(r=$ -0.510; $O R=19.942)$, and hematoma formation $(r=0.722 ; O R=0.015)$.

\section{Discussion}

ESWT has less trauma, low risk, accurate curative effect, and is a good choice for fracture nonunion patients who do not want to undergo surgery. It has shown the ability to induce healing of bone such as tibia, femur, and wrist, et al. in some studies $[16,17]$. In this study, forty-two of 72 fracture nonunion, are grade 3 and 4 healing, accounting for $58 \%$ of the total number of fracture nonunion. This result further proves that ESWT has a better therapeutic effect on nonunion.

However, some patients do not get satisfying efficacy after ESWT and delay time to choose more effective other treatments. In this study, thirty of the seventy-two fracture nonunion have a healing grade $\leq 2$, indicating that doctors need a way to determine early whether ESWT is effective for fracture nonunion. Applying UE, we find that some factors may be correlated to the effect of ESWT on fracture nonunion.

Statistical analysis revealed that nonunion gap was significantly correlated with ESWT outcome $(r=$ $-0.723)$ and was a risk factor in nonunion healing ( $O R=3.074)$ (Table 1 and Table 2$)$. In our study, 18 nonunion had a gap of $>5 \mathrm{~mm}$ and only one (5.5\%) achieved healing of grade 3 . To further investigate the correlation between nonunion gap and outcome, we performed ROC analysis and calculated that the cut-off was $4.200 \mathrm{~mm}$. It meant that the prognosis would be poor if the nonunion gap was $>4.200 \mathrm{~mm}$ (Fig. 1). Previous studies had reported that a nonunion gap of $>5 \mathrm{~mm}$ indicated a poor prognosis with ESWT[8], and our study supported this conclusion. In addition, we found that the results of the nonunion 
gap measured by ultrasound and X-ray were consistent, but ultrasound technology had the advantage of observing the nonunion gap from multiple angles. $[18,19]$.

Local blood supply had a strong effect on bone healing and nonunion frequently occur in fractures with poor blood supply[18, 19], such as in fractures of the scaphoid bone or distal tibia. Our study revealed that good local blood supply was a protective factor in nonunion $(\mathrm{OR}=0.191)$, and was significantly correlated with ESWT outcomes $(r=0.611)$ (Table 1 and Table 2). Moreover, we found that fracture nonunion of the femoral neck and distal tibia had a poor blood supply and weak response to ESWT. In contrast, fracture nonunion of the femur and humerus had an abundant blood supply and showed a strong response to ESWT[18, 19].

ESWT is a form of "mechanotherapy". Impulse energy is conducted to the nonunion callus and periosteum via its "mechano-transduction" effect[20]. The impulse energy causes formation of microfractures and hematoma, and thus reactivates the fracture-healing mechanism[21]. According to this mechanism, we hypothesized that: effective micro-fracture and hematoma formation induced by ESWT was very important; micro-fracture was related to the hardness of callus and impulse energy. ARFI was a technique already in use to estimate the hardness of thyroid nodules or atherosclerotic plaques[22, 23]. Here, we adopted it as an innovative approach to estimating the hardness of callus, and found it practicable. After linear-regression analysis and logistic regression analysis, we found that hardness of callus/impulse energy was a risk factor of ESWT outcome (OR =19.942), and was significantly correlated to outcome ( $r=0.510)$ (Fig. 4). To further analyze the correlation between hardness of callus/impulse energy and ESWT outcome, ROC analysis was used (cut-off point of hardness of callus/impulse energy = 2.555 ) and this revealed that the prognosis was poor if hardness of callus/impulse energy was $>2.555$. Moreover, hematoma formation was also significantly correlated to outcome $(r=0.722)$ and acted as a protective factor of ESWT outcome $(\mathrm{OR}=0.015)$ (Fig. 5).

To eliminate confounding factors, multiple linear regression analysis was performed and this revealed that the four ultrasound signs correlated significantly with ESWT outcome, with a significance rank of: fracture nonunion gap $(t=-5.224)>$ hematoma formation $(t=4.288)>$ local blood supply $(t=3.268)>$ hardness of callus/impulse energy $(t=-2.056)$. The above results indicated that some factors might corelated to the clinical efficacy of ESWT on fracture nonunion.

Our study still has several limitations. Firstly, the sample size of the present study is insufficient, which may be correlated to nonunion morbidity; secondly, there is currently no objective standard to identify local blood supply and the outcome of nonunion, which means our standard depends on the clinical experience of the doctor and may be subjective. The further expanded sample size and the unified standards in clinical can help to confirm the conclusion.

In summary, Nonunion gap larger than $4.200 \mathrm{~mm}$ and hardness of callus/impulse energy larger than 2.555 are risk factors of ESWT outcome, and good local blood supply and effective hematoma formation induced by ESWT are protective factors. By analyzing the related factors of extracorporeal shockwave 
therapy on fracture nonunion, clinicians may screen out the patients with fracture nonunion who are not suitable for ESWT, and reduce the risk of fracture nonunion.

\section{Abbreviations}

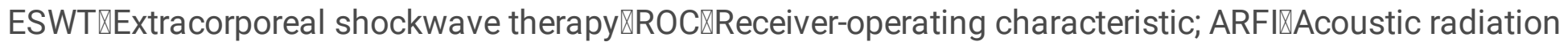
force impulse区UE: Ultrasound Examination

\section{Declarations}

\section{Acknowledgements}

Not applicable.

\section{Authors' contributions}

Xiaolin Li, Lixin Jiang, Jinghuan Huang and Shiyang Yu gave contributions to study design and revise the article, Ang Li and Rui Wang gave contributions to collect and analyse data, draft the article. All authors discussed the results and contributed to the final version of the manuscript. The authors read and approved the final manuscript.

\section{Funding}

This study has received funding by Natural Science Foundation of China Grant No. 81572178 and No. 82072422.

\section{Availability of data and materials}

The datasets used or analyzed during the current study are available from the corresponding author on reasonable request.

\section{Ethics approval and consent to participate}

The study was approved by the Ethics Committees of Shanghai sixth people's hospital. All procedures were carried out in accordance with ethical standards. Written informed consent was obtained from all individual participants included in the study. 


\section{Consent for publication}

All participants gave written and informed consent for publication for all personal or clinical details along with any identifying images to be published in this study.

\section{Competing interest}

The authors have no conflicts of interest to declare.

\section{Author details}

1Department of Orthopaedic Surgery, Shanghai Jiao Tong University Affiliated Sixth People's Hospital, 600 Yishan Rd, Shanghai, PR China.

2Department of Ultrasound in Medicine, Shanghai Jiao Tong University Affiliated Sixth People's Hospital, ,600 Yishan Rd, Shanghai Institute of Ultrasound in Medicine, Shanghai, PR China.

\section{References}

1. Elster EA, Stojadinovic A, Forsberg J, Shawen S, Andersen RC, Schaden W. Extracorporeal Shock Wave Therapy for Nonunion of the Tibia. Journal of Orthopaedic Trauma. 2010;24:133-41.

2. Kobayashi M, Chijimatsu R, Yoshikawa H, Yoshida K. Extracorporeal shock wave therapy accelerates endochondral ossification and fracture healing in a rat femur delayed-union model. Biochemical and Biophysical Research Communications. 2020;530:632-7.

3. Reilly JM, Bluman E, Tenforde AS. Effect of Shockwave Treatment for Management of Upper and Lower Extremity Musculoskeletal Conditions: A Narrative Review. PM and R. 2018;10:1385-403.

4. Saunier J, Chapurlat R. Stress fracture in athletes. Joint Bone Spine. 2018;85:307-10.

5. Großner T, Schmidmaier G. Konservative Therapieoptionen der Pseudarthrosen. Der Unfallchirurg. 2020;123:705-10.

6. Willems A, Van Der Jagt OP, Meuffels DE. Extracorporeal Shock Wave Treatment for Delayed Union and Nonunion Fractures: A Systematic Review. Journal of Orthopaedic Trauma. 2019;33:97-103.

7. Haffner N, Antonic V, Smolen D, Slezak P, Schaden W, Mittermayr R, et al. Extracorporeal shockwave therapy (ESWT) ameliorates healing of tibial fracture non-union unresponsive to conventional therapy. Injury. 2016;47:1506-13.

8. Alkhawashki HMI. Shock wave therapy of fracture nonunion. Injury. 2015;46:2248-52.

9. Palanisamy P, Alam M, Li S, Chow SKH, Zheng Y. Low-Intensity Pulsed Ultrasound Stimulation for Bone Fractures Healing. Journal of Ultrasound in Medicine. 2021;:jum.15738. 
10. Hryhorczuk AL, Restrepo R, Lee EY. Pediatric Musculoskeletal Ultrasound: Practical Imaging Approach.

http://dx.doi.org/102214/AJR1515858

. 2016;206:W62-72. doi:10.2214/AJR.15.15858.

11. Kotlarsky P, Feldman O, Shavit I, Eidelman M. The use of real-time sonography-assisted fracture reduction in children with displaced forearm fractures. Journal of Pediatric Orthopaedics $B$. 2021;2021. doi:10.1097/BPB.0000000000000886.

12. Cacchio A, Giordano L, Colafarina O, Rompe JD, Tavernese E, loppolo F, et al. Extracorporeal ShockWave Therapy Compared with Surgery for Hypertrophic Long-Bone Nonunions. The Journal of Bone \& Joint Surgery. 2009;91:2589-97.

13. Kertzman P, Császár NBM, Furia JP, Schmitz C. Radial extracorporeal shock wave therapy is efficient and safe in the treatment of fracture nonunions of superficial bones: A retrospective case series. Journal of Orthopaedic Surgery and Research. 2017;12.

14. Yeh $\mathrm{C}-\mathrm{C}$, Horng $\mathrm{H}-\mathrm{C}$, Wang $\mathrm{P}-\mathrm{H}$. Acoustic radiation force imaging (ARFI): A new powerful tool of ultrasound. Journal of the Chinese Medical Association. 2017;80:681-2.

15. Choi YS, Kim KS. Plate augmentation leaving the nail in situ and bone grafting for non-union of femoral shaft fractures. International Orthopaedics. 2005;29:287-90.

16. Sandoval C, Valenzuela Á, Rojas C, Brañes M, Guiloff L. Extracorporeal shockwave therapy for atrophic and oligotrophic nonunion of tibia and femur in high energy trauma patients. Case series. International Journal of Surgery Open. 2017;9:36-40.

17. Quadlbauer S, Pezzei C, Beer T, Jurkowitsch J, Keuchel T, Schlintner C, et al. Treatment of scaphoid waist nonunion by one, two headless compression screws or plate with or without additional extracorporeal shockwave therapy. Archives of Orthopaedic and Trauma Surgery. 2019;139:281-93.

18. Gallardo-Calero I, Barrera-Ochoa S, Manzanares MC, Sallent A, Vicente M, López-Fernández A, et al. Vascularized periosteal flaps accelerate osteointegration and revascularization of allografts in rats. Clinical Orthopaedics and Related Research. 2019;477:741-55.

19. Cheema HS, Cheema AN. Radiographic evaluation of vascularity in scaphoid nonunions: A review. World journal of orthopedics. 2020;11:475-82.

20. d'Agostino MC, Craig K, Tibalt E, Respizzi S. Shock wave as biological therapeutic tool: From mechanical stimulation to recovery and healing, through mechanotransduction. International Journal of Surgery. 2015;24:147-53.

21. Gerdesmeyer L, Schaden W, Besch L, Stukenberg M, Doerner L, Muehlhofer H, et al. Osteogenetic effect of extracorporeal shock waves in human. International Journal of Surgery. 2015;24 Pt B:1159.

22. Czernuszewicz TJ, Homeister JW, Caughey MC, Wang Y, Zhu H, Huang BY, et al. Performance of acoustic radiation force impulse ultrasound imaging for carotid plaque characterization with histologic validation. Journal of Vascular Surgery. 2017;66:1749-1757.e3. 
23. Doherty JR, Dahl JJ, Kranz PG, El Husseini N, Chang HC, Chen NK, et al. Comparison of Acoustic Radiation Force Impulse Imaging Derived Carotid Plaque Stiffness with Spatially Registered MRI Determined Composition. IEEE Transactions on Medical Imaging. 2015;34:2354-65.

\section{Figures}

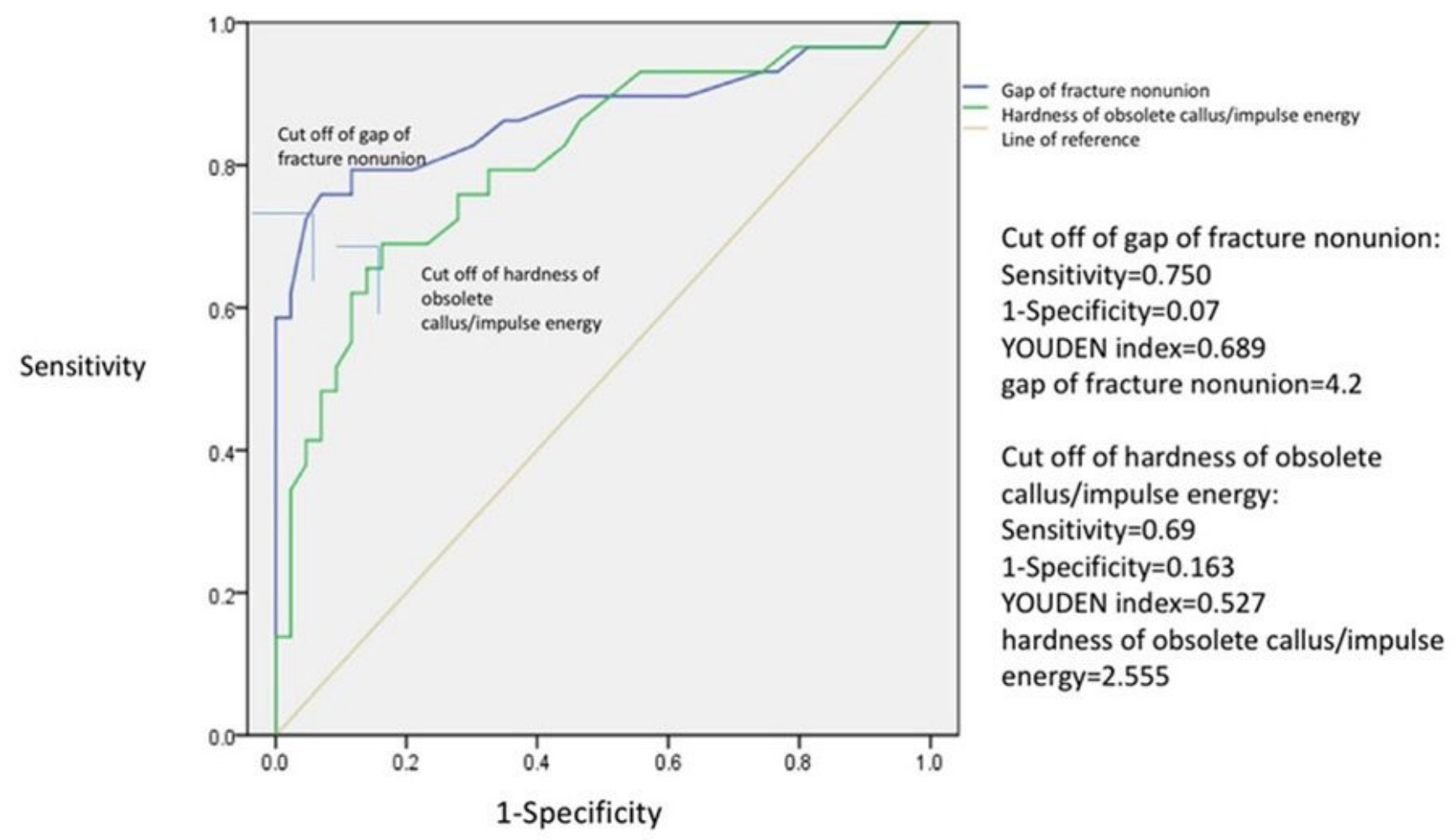

\section{Figure 1}

ROC test of cut off of gap of fracture non-unions and hardness of callus/impulse energy. 


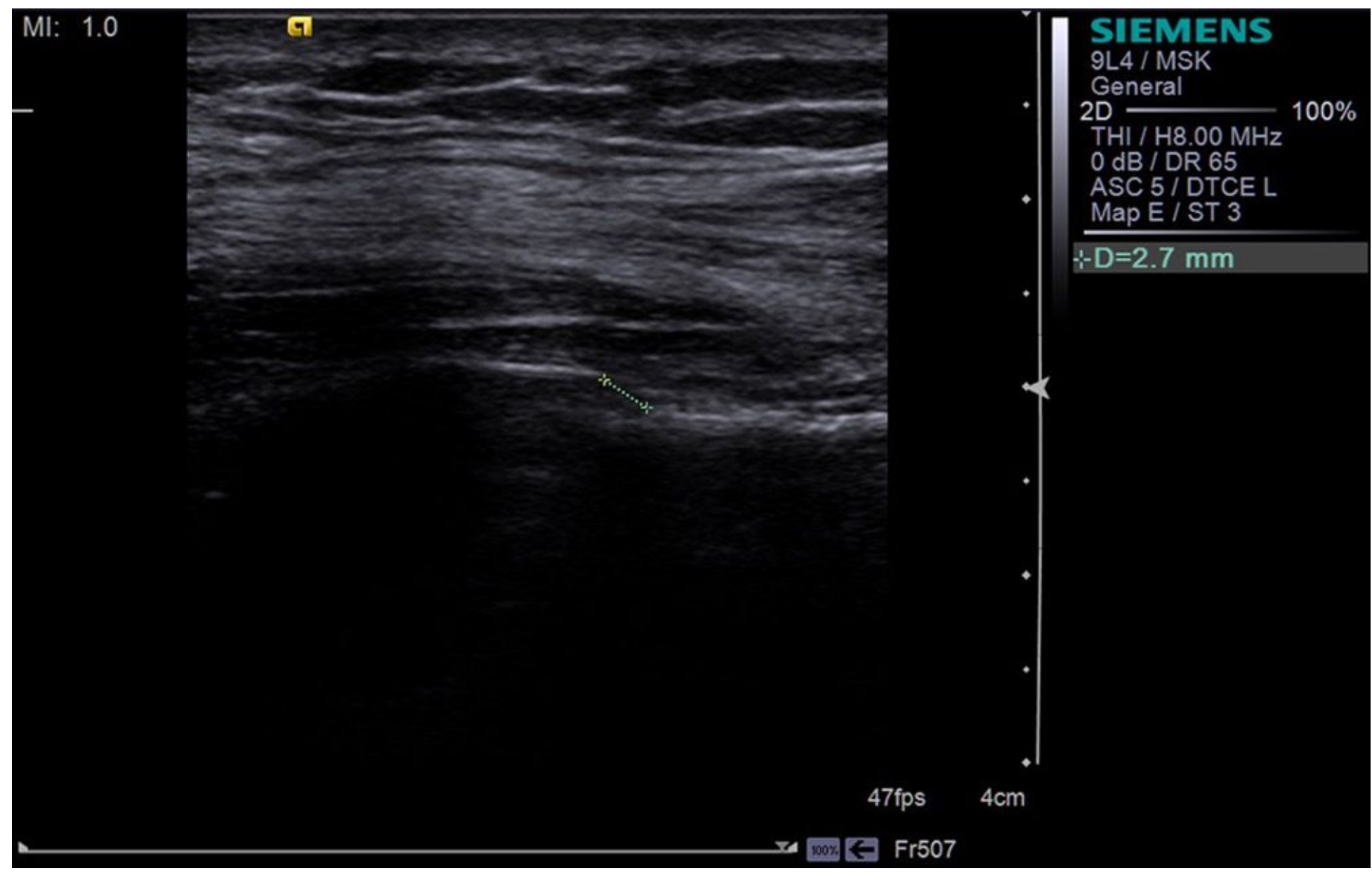

Figure 2

Ultrasound for nonunion gap measuring (unit: $\mathrm{mm}$ ). 

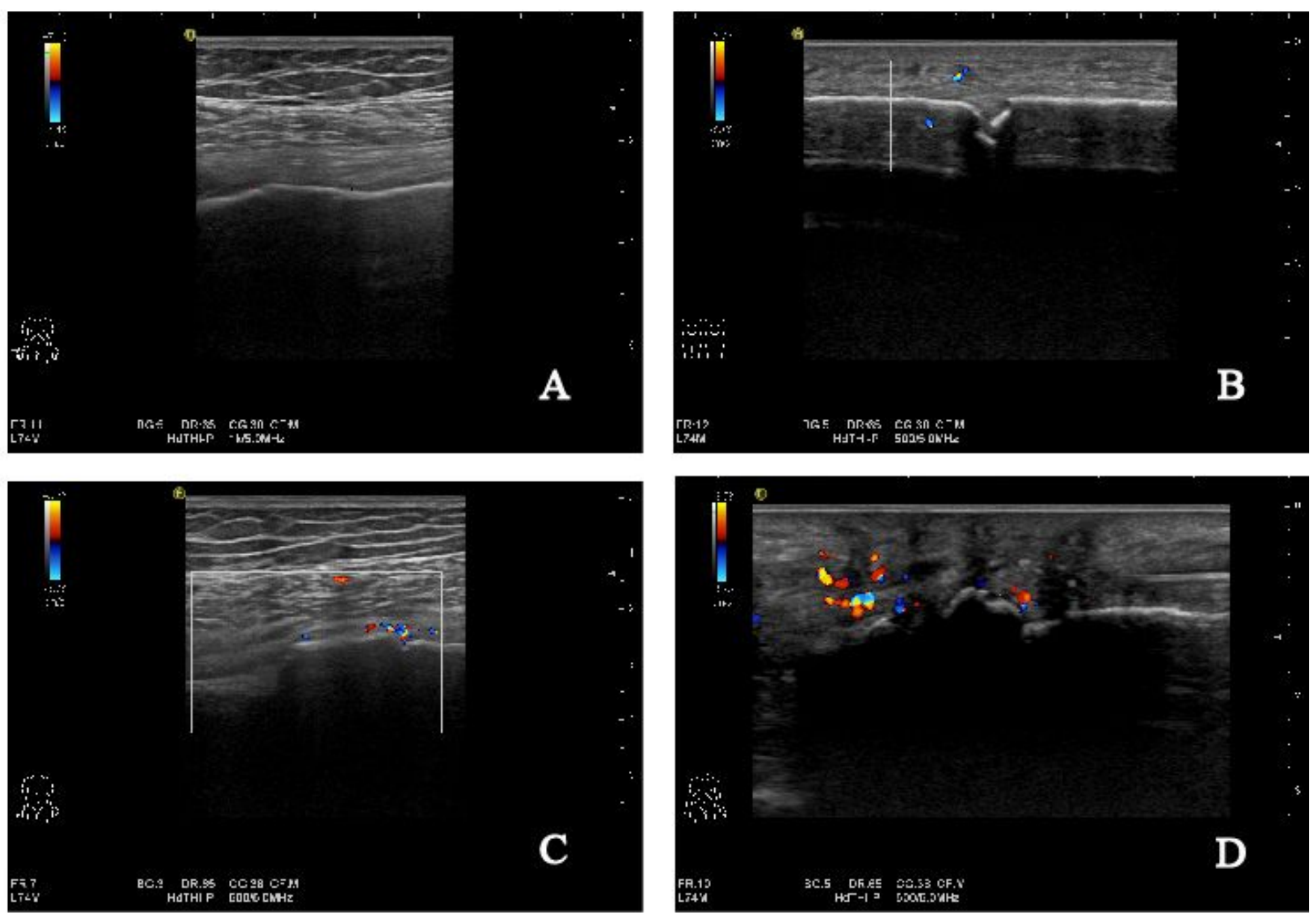

\section{Figure 3}

Ultrasound for local blood supply detecting (A: grade1 B: grade2 C: grade3 D: grade4). 


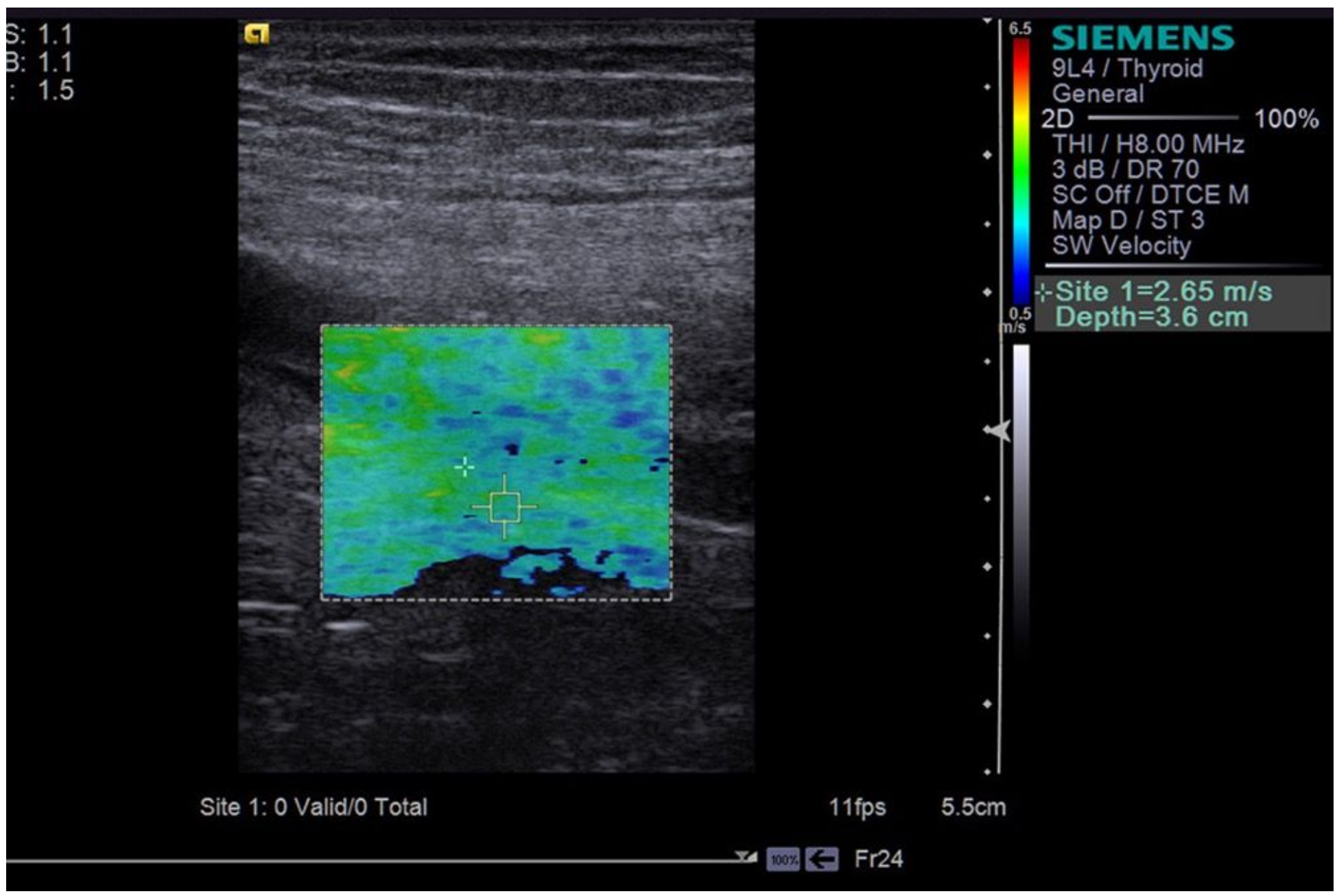

Figure 4

ARFI for measuring hardness of callus (units: $\mathrm{m} / \mathrm{s}$ and $\mathrm{cm}$ ). 

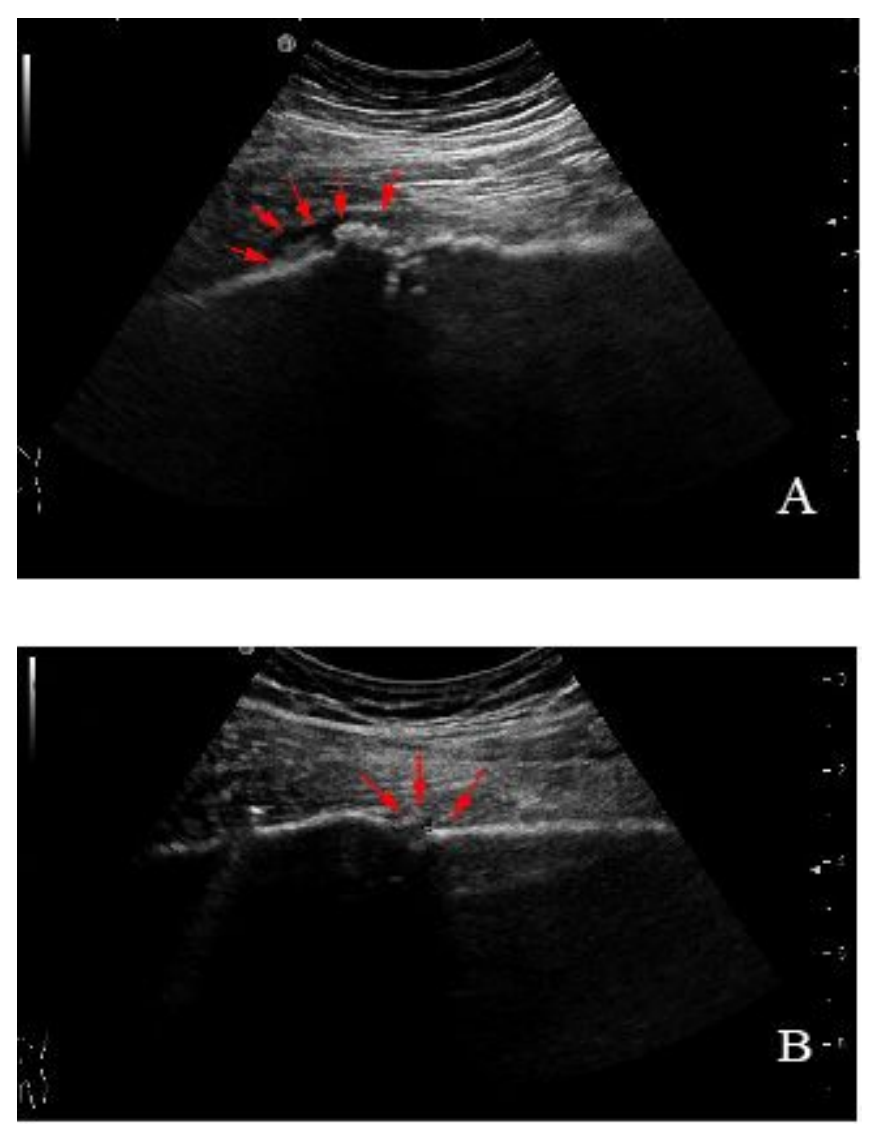

\section{Figure 5}

Ultrasound for hematoma detecting (A: hematoma formation B: non-hematoma formation). The red arrows show the locations of hematoma and non-hematoma formation.

\section{Supplementary Files}

This is a list of supplementary files associated with this preprint. Click to download.

- S.1.tif

- S.2.tif

- S.3.tif

- S.4.tif

- S.5.tif 